\title{
Influence on Visibility Due to Differences of Guide Wire Movement-Visual \& Physical Evaluations
}

\author{
Hisaya Sato ${ }^{1,2 *}$, Daisuke Kittaka², Miwa Ohsawa3 ${ }^{3}$, Kyoichi Kato ${ }^{1,4}$ \\ ${ }^{1}$ Graduate School of Health Sciences, Showa University, Tokyo, Japan \\ ${ }^{2}$ Department of Radiological Technology, Showa University Hospital, Tokyo, Japan \\ ${ }^{3}$ Department of Radiological Technology, Showa University Fujigaoka Hospital, Yokohama, Japan \\ ${ }^{4}$ Department of Unification Radiological Technology, Showa University, Tokyo, Japan \\ Email: ^hisaya@cmed.showa-u.ac.jp
}

How to cite this paper: Sato, H., Kittaka, D., Ohsawa, M. and Kato, K. (2020) Influence on Visibility Due to Differences of Guide Wire Movement-Visual \& Physical Evaluations. Open Journal of Applied Sciences, 10, 577-591.

https://doi.org/10.4236/ojapps.2020.101004 $\underline{1}$

Received: September 1, 2020

Accepted: October 6, 2020

Published: October 9, 2020

Copyright (c) 2020 by author(s) and Scientific Research Publishing Inc. This work is licensed under the Creative Commons Attribution International License (CC BY 4.0).

http://creativecommons.org/licenses/by/4.0/

\begin{abstract}
The visibility of moving images during cardiac catheterization and treatment may be reduced by a number of factors. First, it involves multiple movements that occur simultaneously, such as the movements due to the heart beat and movement of the guide wire used during the treatment. There is also the influence of the X-ray dose on the image quality in the X-ray output. If X-rays are irradiated onto moving objects such as a guide wire moving during treatment of the heart, cardiac catheterization may be displaced to the next image recorded even when an insufficient X-ray dose has been irradiated because the imaged object is moving during the time the $\mathrm{X}$-rays are emitted (pulse width). If the X-ray dose planned to be irradiated to the target is low, there is also the possibility that noise will appear in the image, and the imaged object may be lost in noise and visibility be reduced. For this reason, we conducted basic research into how changes in the speed of rotation of guide wires affect visibility when wires are positioned horizontally and vertically, using a dynamic phantom and recorded X-ray moving images. The purpose of this study is to elucidate whether the deterioration in the visibility is affected by the X-ray dose, the orientation or movement of a guide wire, or caused by other conditions, in order to contribute to improving the visibility in the $\mathrm{X}$-ray moving images. The results showed a lower visual evaluation only in the vertical direction at the more rapid movement here, but this did not result in significant changes in the physical evaluation. This suggests that the structure and characteristics of the human eyes would be involved, as human vision is stronger with lateral movements and weaker with vertical movements due to the arrangement of the human eyes, side by side. Findings from this
\end{abstract}


basic study can be utilized to improve the visibility in the X-ray moving images by paying attention to the observation environment of the observer of the X-ray moving images. In addition, the findings of this study can also be used to determine protocols for improving visibility in X-ray moving images, such as adjusting the X-ray dose in an X-ray device when further improvement is required. Therefore, this study was able to provide suggestions to contribute to the development of improved visibility of X-ray moving images.

\section{Keywords}

X-Ray Moving Images, Visibility, Human Vision, Guide Wire

\section{Introduction}

Cardiac catheterization that involves the heart movement is performed with multiple images recorded in a short time. This is because it is necessary to collect moving images to obtain images that are sufficiently accurate to depict the heart movement including the changes in the movement of the heart walls, in the blood flow to the coronary arteries, the blood vessels feeding nutrition to the heart, and in the morphology of the blood vessels obtained by using shadow images of the blood flow. In cardiac catheterization, many tools including guide wires, balloons, and stents are used in the treatment. Blood vessels subject to treatment involve the main coronary artery, which is less than $5 \mathrm{~mm}$ in diameter and the $2 \mathrm{~mm}$ diameter lateral branches.

Cardiac catheterization is conducted by moving and controlling a guide wire in all directions: vertical, horizontal, and diagonal depending on the movement of the blood vessels. Because visibility is particularly important in the operation of the guide wire during the treatment and it is necessary to ensure high quality images providing clear visibility. However, during cardiac catheterization and treatment problems due to poor visibility may be experienced.

The visibility of moving images during cardiac catheterization and treatment may be reduced by a number of factors. First, it involves multiple movements that occur simultaneously, such as the movements due to the heart beat and movement of the guide wire used during the treatment. There is also the influence of the X-ray dose on the image quality in the X-ray output.

If $\mathrm{X}$-rays are irradiated onto moving objects such as a guide wire moving during treatment of the heart, cardiac catheterization may be displaced to the next recorded image even when an insufficient X-ray dose has been irradiated because the imaged object is moving during the time the X-rays are emitted (pulse width). If the X-ray dose planned to be irradiated to the target is low, there is also the possibility that noise will appear in the image, and the imaged object may be lost in noise and visibility be reduced.

These above conditions are considered to be factors that cause poor visibility in moving images. There are many studies that evaluate visibility in X-ray moving 
images using a static phantom. However, these studies focus on organs, blood flow, and blood vessels that are constantly moving, including therapeutic instruments. With a static phantom there are limitations in the evaluation of visibility because the movement of the evaluation target is not taken into consideration.

It is important to evaluate the visibility using a dynamic phantom as in this study. Despite being at the limit of visibility evaluation, one of the reasons why visibility evaluations are performed by static phantoms is that there are few dynamic phantoms used as evaluation tools and as the simulation of the heart (anatomical scatterers and also possibly one of the causes arises from the dynamic phantom that has been overlaid) has not been realized. For this reason, we conducted basic research into how changes in the speed of rotation of guide wires affect visibility when wires are positioned horizontally and vertically, using a dynamic phantom and recorded X-ray moving images. The purpose of this study is to elucidate whether the deterioration in the visibility is affected by the $\mathrm{X}$-ray dose, the orientation or movement of a guide wire, or caused by other conditions, in order to contribute to improving the visibility in the X-ray moving images.

\section{Methods}

\subsection{Equipment}

This study used a cardiovascular X-ray apparatus, Allura Xper FD 10/10 manufactured by Phillips. For the moving image viewer, a kada-View manufactured by Photron was used. The image analysis software ImageJ was used with two 10 $\mathrm{cm}$ Acrylic phantoms as the imaged objects. For the visual and physical evaluations, a KS dynamic phantom (Figure 1) manufactured by Kyoto Kagaku Co. was used. To ensure high-contrast signals the following guide wires for clinical use were used: 0.009 inch (X-treme PV), 0.010 inch (Decillion), and 0.014 inch (Tresure XS), manufactured by Asahi Intec Co., Ltd. The statistical analysis processing software was JMP 1.4.

\subsection{The KS Dynamic Phantom for Medical X-Ray Moving Images by Kyoto Kagaku Co}

The KS dynamic phantom was set so that it could operate at 3.0 or $6.0 \mathrm{rpm}$, and the guide wire was set at the position shown in Figure 2(a) to ensure that the guide wire was vertical or horizontal to the diameter of the KS phantom. The center of the guide wire was set at $60 \mathrm{~mm}$ from the center of the KS dynamic phantom. The dynamic speed at this position is $18.8 \mathrm{~mm} / \mathrm{sec}$ at $3.0 \mathrm{rpm}$, and when converted to heartbeats equivalent to 57 beats/min (Figure 2(b)), at 6.0 $\mathrm{rpm}$, the speed is twice that at $3.0 \mathrm{rpm}[1]$.

\subsection{Imaging Conditions of the Cardiovascular X-Ray Apparatus}

Imaging was performed under conditions like those of clinical settings. The image acquisition geometry is shown in Figure 3. 
Two $10 \mathrm{~cm}$ thick acrylic sheets were used as the thickness of the imaged object, and the KS dynamic phantom was set between them. The image acquisition angle was set to the Anterior-Posterior (AP) direction. Table 1 and Table 2
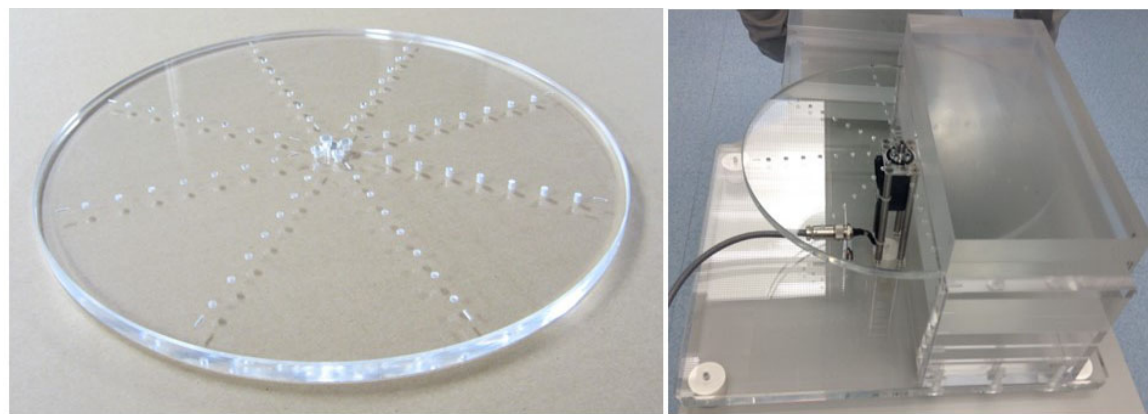

Sato, H. et al., Journal of Applied Clinical Medical Physics. 2015; 16(2).

Figure 1. Photographs of the KS dynamic phantom.

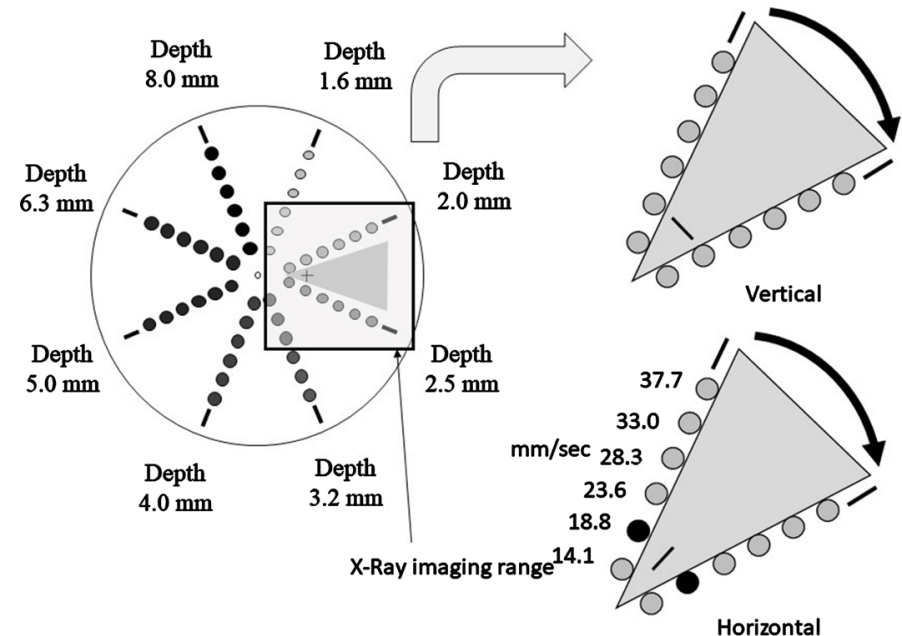

(a)

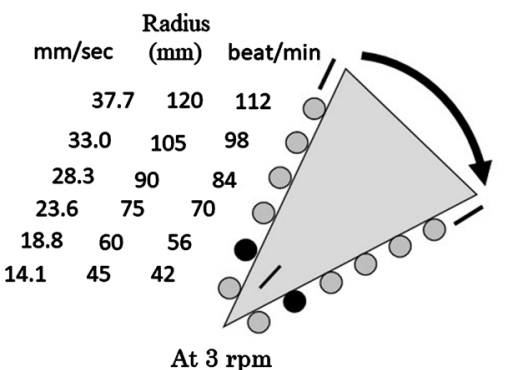

(b)

Positional information and speeds when the KS dynamic phantom is rotated at $3.0 \mathrm{rpm}$

Figure 2. (a) Structure of the KS dynamic phantom and details of guide wire placement; (b) Positions and speeds of guide wire locations.

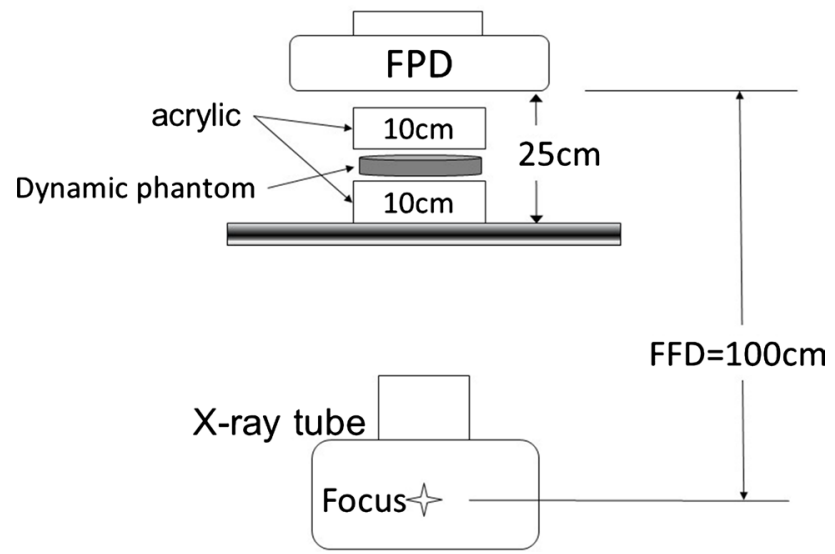

Figure 3. Geometry of moving image acquisition arrangement. 
Table 1. Types of by cardiovascular X-ray apparatus, digital, and load filters that were used in the automatically processing during the imaging.

\begin{tabular}{ccc}
\hline Apparatus & Imaging & Additional filters \\
\hline & Harmonization & \\
Allura Xper FD10/10 & Rounding & None \\
& Edge enhancement & \\
& Brightness Imaging & \\
&
\end{tabular}

Table 2. Cardiovascular X-ray apparatus and imaging conditions.

\begin{tabular}{ccccc}
\hline \multicolumn{5}{c}{ Imaging } \\
\hline Apparatus & Tube voltage $(\mathrm{kV})$ & Tube current $(\mathrm{mA})$ & Pulse width $(\mathrm{msec})$ & Frame rate $(\mathrm{F} / \mathrm{S})$ \\
\hline Allura Xper FD10/10 & 81.0 & 200.0 & 5.0 & 7.5
\end{tabular}

show the imaging conditions and the types of digital filters that are automatically processed during imaging.

Imaging was conducted using an 8-inch X-ray detector $(720 \times 720$-pixel, 14 bit), and image data were output as $1024 \times 102410 \mathrm{bit}$. The transfer rate from the detector to the image processing PC was set to $9.5 \mathrm{~ms}$. As the digital filter, we did not use recursive filters because they emphasize the lag. Images were processed under the following conditions: Harmonization: 51\%, Rounding factor: 12.5\%, Edge enhancement: 7, and Brightness: 0.06 .

\subsection{Visual Evaluation with Guide Wires Set at the Different Orientations and Speeds}

For imaged objects, we used a radio-opaque portion of $20 \mathrm{~mm}$ from the tip of the $0.009,0.010$, and 0.014 -inch guide wires, which are used clinically in cardiovascular treatment. For the visual evaluation, the KS dynamic phantom was moved at 3.0 or $6.0 \mathrm{rpm}$. Figure 2(a) shows how the guide wires of all the sizes set horizontally and vertically moved in the X-ray imaging range. We evaluated whether the direction and speed of movement affect the visibility. The visibility evaluation was performed by five physicians experienced with X-ray moving images in daily clinical practice, each having taken part in more than 300 coronary artery treatments. The quality of the guide wire images was scored based on the evaluation criteria described below, and the average score of the 5 physicians was calculated. The reference image in inches of each guide wire was a static image. To perform visual evaluations of the 0.009 -inch guide wire at the static state and that moving at $3.0 \mathrm{rpm}$, a 0.009 -inch guide wire was set vertically in the static state and rotated at $3.0 \mathrm{rpm}$ in the vertical direction.

Next, we evaluated a static image of the same guide wire and the image rotating at $6.0 \mathrm{rpm}$. The visual evaluation results at 3.0 and $6.0 \mathrm{rpm}$ were compared, and 0.010- and 0.014-inch guide wires were also evaluated in the same manner. Further, the guide wire moving in the horizontal direction was visually evaluated in the same manner as the guide wire rotating in the vertical direction. Evalua- 
tion criteria: compared to static images, they are clearly visible (equivalent to the static image): 5 points; Visible (could be compared): 4 points; Borderline visible but can be compared: 3 points; Hard to see (could be compared but only poorly): 2 points; and Invisible (could not be compared): 1 point.

\subsection{Physical Evaluation with Guide Wires Set at the Different Orientations and Speeds}

Based on the images used for the visual evaluation, the profile curves of the guide wires [2] were calculated using ImageJ. To unify the measurement points, the profile curve at the center of the vertical and horizontal guide wires was calculated as shown in Figure 4. To reduce the measurement error, the same part was measured 5 times and the average value of the measurements was calculated. In the physical evaluation using the profile curve, the difference between the background and the signal value was evaluated by the method shown in Figure 5. Further, taking differences by measurers into account, these measurements

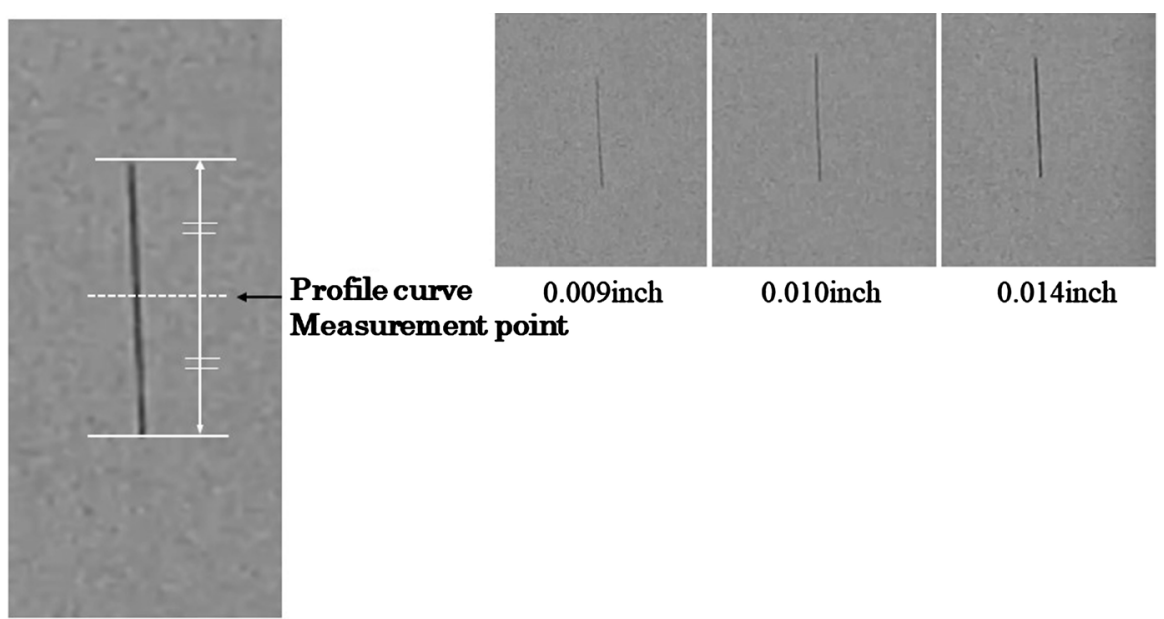

Figure 4. Measurement points on profile curves of the guide wire.

Gray value

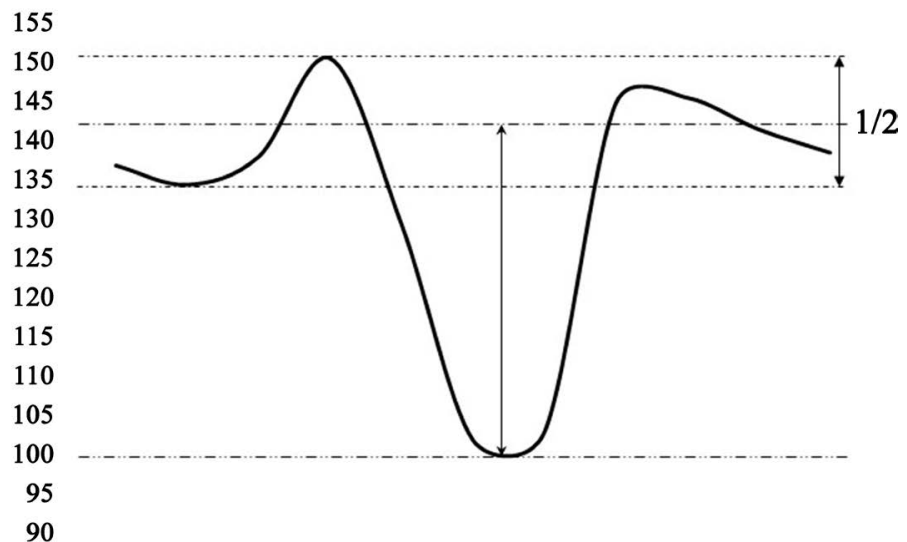

KS dynamic phantom speed: 6.0 rpm; Guide wire movement: Horizontal; Guide wire diameter: 0.014 inch.

Figure 5. Details of calculation of the difference between background and signal values. 
were performed by one radiological technologist with 26 years of experience and accredited by the Japanese professional accreditation board of radiological technologist for angiography and intervention.

\subsection{Evaluation of Background Noise with Guide Wires Moving at the Different Speeds}

Using images of a 0.009-inch guide wire, a profile curve was calculated for the range from $5 \mathrm{~mm}$ to the left and right of the center of the vertically set guide wires (Figure 4), and other parts of the guide wires were included in the background noise (Figure 6). Further, we performed an evaluation to establish that there was an absence of influence of speed on the background noise.

\section{Results}

\subsection{Visual Evaluation with Guide Wires Set at the Different Orientations and Speeds}

Figure 7 shows the results of the visual evaluations of the guide wires of all the three wire diameters investigated here set horizontally and vertically, with the KS dynamic phantom rotated at 3.0 and $6.0 \mathrm{rpm}$. With the 0.014 -inch horizontal guide wire, the visual rating was 5.0 points at both the 3.0 and $6.0 \mathrm{rpm}$ speeds. With the 0.01 -inch wire, the averaged visual rating was 4.8 points at $3.0 \mathrm{rpm}$, and 4.4 points at $6.0 \mathrm{rpm}$. With the 0.009 -inch wire, the rating was 4.6 points at $3.0 \mathrm{rpm}$ and 3.6 points at $6.0 \mathrm{rpm}$. With the 0.014 -inch vertical guide wire, the visual rating was 5.0 points at $3.0 \mathrm{rpm}$ and 3.2 points at $6.0 \mathrm{rpm}$. With the 0.01 -inch wire, the rating was 4.0 points at $3.0 \mathrm{rpm}$ and 2.2 points at $6.0 \mathrm{rpm}$. With the 0.009 -inch wire, it was 3.6 points at $3.0 \mathrm{rpm}$ and 2.0 points at $6.0 \mathrm{rpm}$. As described above, in the movements with the KS dynamic phantom the visual evaluations of both the horizontally and vertically positioned wires 3.0 and 6.0 $\mathrm{rpm}$ showed increasingly higher scores as the guide wire thickness increased.

\section{Gray value}

160

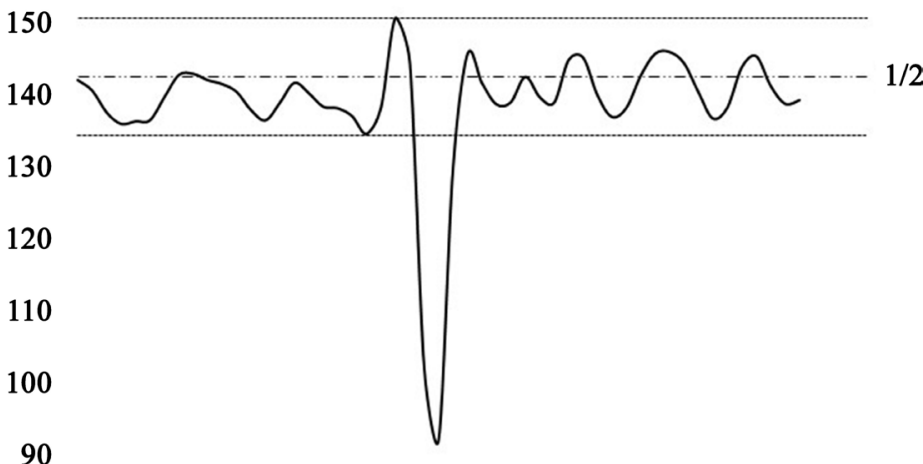

90

80

KS dynamic phantom speed: 6.0 rpm; Guide wire movement: Horizontal; Guide wire diameter: 0.014 inch

Figure 6. Details of calculation of background noise. 


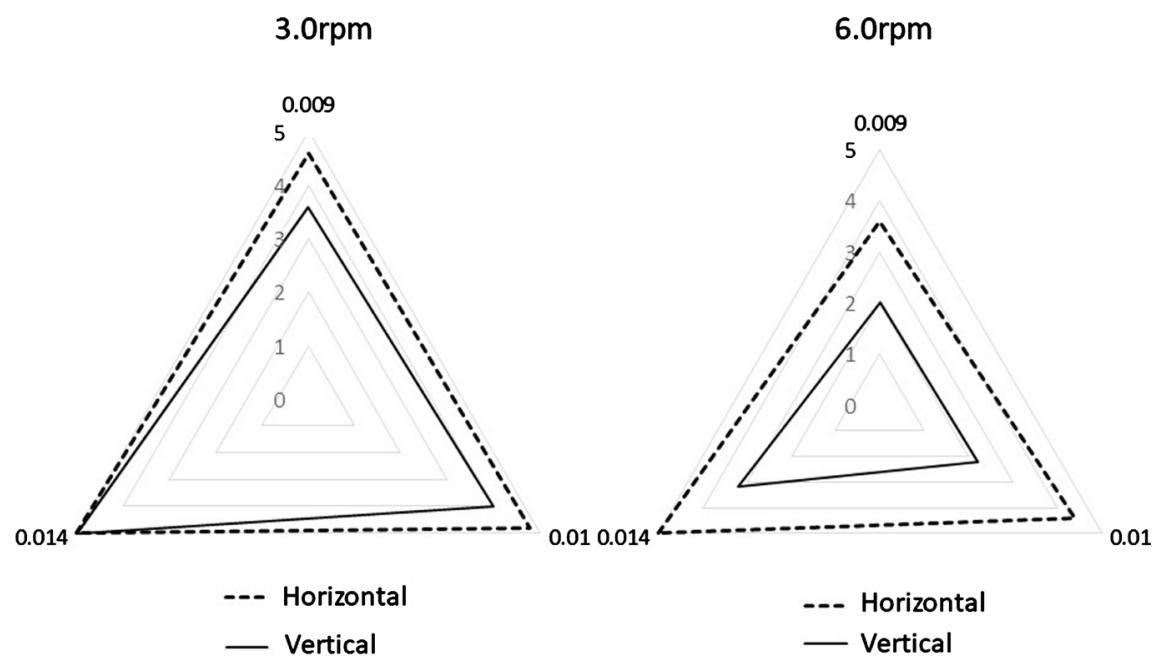

Figure 7. Results of the visual evaluation of guide wires by diameter set horizontally and vertically.

\subsection{Physical Evaluation with Guide Wires Set at the Different Orientations and Speeds}

Figure 8 shows profile curves of the results of the physical evaluation of the guide wires of all the sizes set horizontally and vertically with the KS dynamic phantom rotated at 3.0 and $6.0 \mathrm{rpm}$. With the 0.014-inch, 0.01-inch, and 0.009 -inch horizontal guide wires, the profile curves showed similar changes at 3.0 and $6.0 \mathrm{rpm}$. The gray values [3] showed lower values at $3.0 \mathrm{rpm}$ than at 6.0 rpm for all thicknesses. With the 0.014-inch guide wire, the gray values were 99.5 and 101.9 at 3.0 and $6.0 \mathrm{rpm}$, respectively; with the 0.01 -guide wire, the grey values were 113.4 and 115.9 at 3.0 and $6.0 \mathrm{rpm}$, respectively; and with the 0.009 -inch guide wire, the grey values were 121.4 and 123.4 at 3.0 and $6.0 \mathrm{rpm}$, respectively.

With the vertical guide wires, both the profile curves and the gray values showed similar tendencies as the horizontal guide wires. With the 0.014-inch guide wire, the gray values were 93.5 and 96.7 at 3.0 and $6.0 \mathrm{rpm}$, respectively; with the 0.01 -guide wire, the values were 115.0 and 117.4 at 3.0 and $6.0 \mathrm{rpm}$, respectively; and with the 0.009 -inch guide wire, they were 120.5 and 120.6 at 3.0 and $6.0 \mathrm{rpm}$, respectively. As described above, with the KS dynamic phantom both the horizontally and vertically positioned wires resulted in gray values at $3.0 \mathrm{rpm}$ that were lower than those at $6.0 \mathrm{rpm}$, and the differences in grey values became larger with thicker guide wires.

\subsection{Evaluation of Background Noise with Guide Wires Moving at the Different Speeds}

Figure 9 shows profile curves of the results of the background noise evaluations of the 0.009-inch guide wire set vertically where the KS dynamic phantom was rotated at 3.0 and $6.0 \mathrm{rpm}$. The range of the profile curves is $5 \mathrm{~mm}$ to the left and right of the center of the guide wire (Figure 9). With the 0.009-inch wires set 
gray value

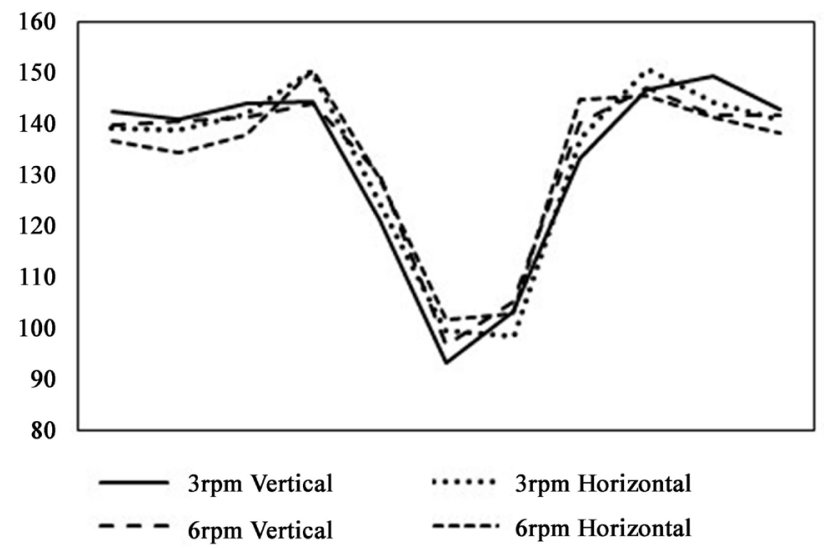

gray value

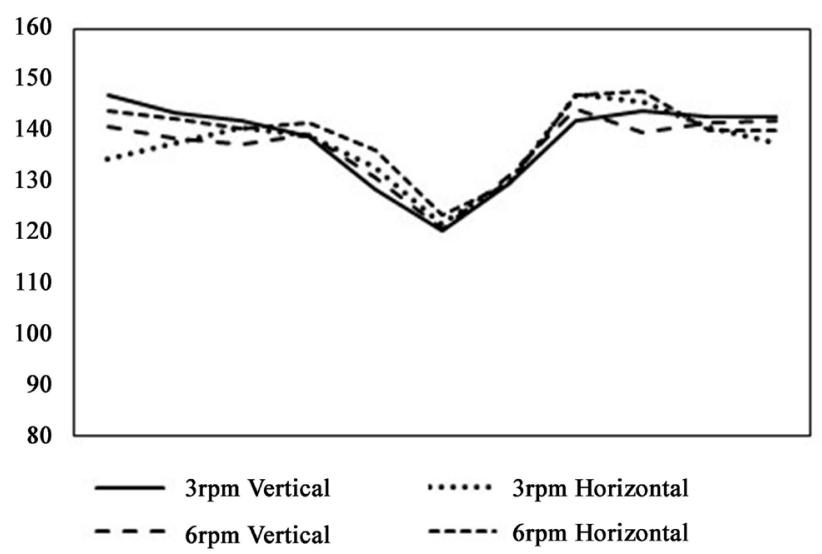

gray value

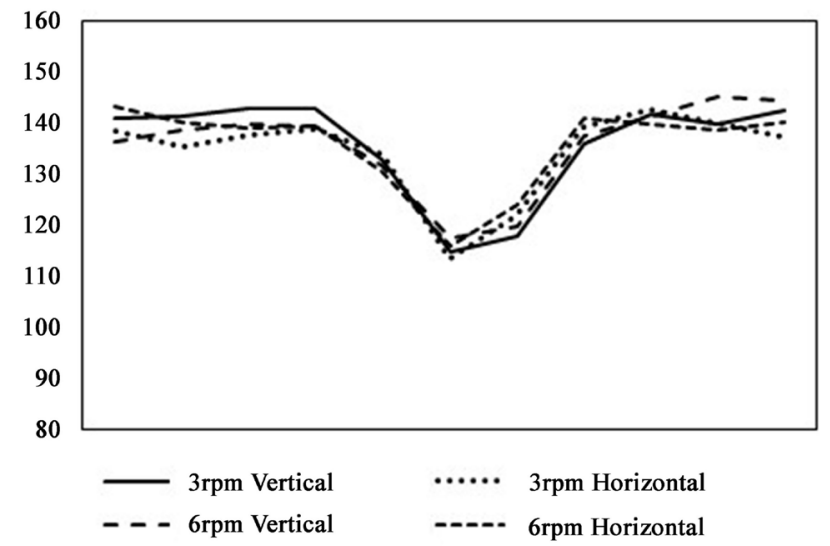

Figure 8. Results of the physical evaluation of guide wires by diameter set horizontally and vertically.

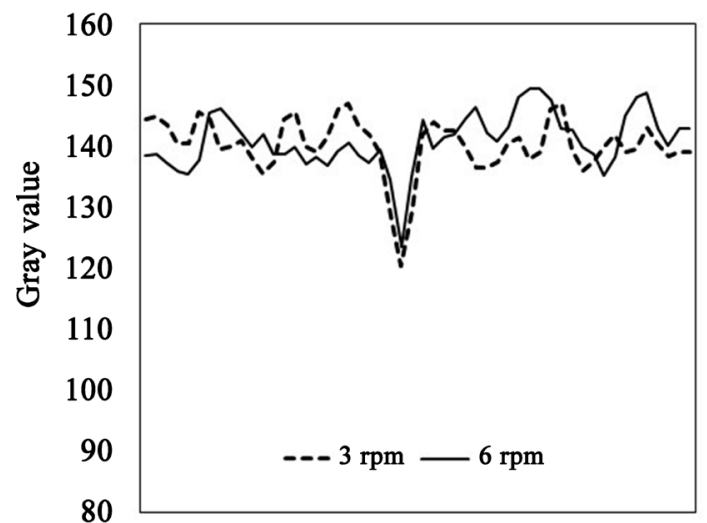

Results at $3.0 \mathrm{rpm}$. Guide wire diameters as in bottom right grid.

Figure 9. Results of the physical evaluation of the background noise of guide wires at 3.0 and $6.0 \mathrm{rpm}$ The 0.009 -inch guide wires here set vertically.

vertically, the maximum, minimum, and median values of the noise in the profile curve at $5 \mathrm{~mm}$ to the left and right of the center of the guide wire were 147.0, 135.5 , and 140.5 at $3.0 \mathrm{rpm}$, and at $6.0 \mathrm{rpm}$ the values were $149.4,135.3$, and 140.7. 


\section{Discussion}

\subsection{Visual Evaluation with Guide Wires Set at the Different Orientations and Speeds}

When horizontal and vertical guide wires were rotated at 3.0 or $6.0 \mathrm{rpm}$, the visual evaluation scores became higher as the guide wire thickness increased. The scores with guide wires set vertically were lower than those set horizontally. In the visual evaluations (Figure 10), there were statistically significant differences between the scores in the different orientations of the guide wires $(\mathrm{P}<$ $0.05)$, and the visual evaluations of guide wires set vertically were statistically significantly smaller. As indicated in Figure 11, there were no statistically significant

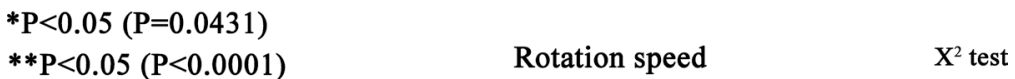

$\mathrm{X}^{2}$ test

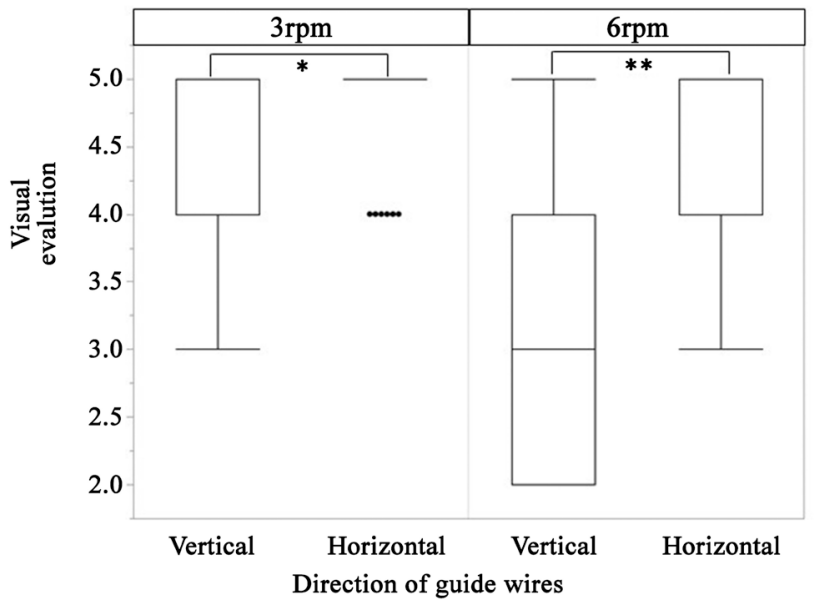

Results of $\mathrm{X}^{2}$ test using statistical analysis processing software Jamp 1.4.

Figure 10. Results of the physical evaluation of the background noise of guide wires at 3.0 and $6.0 \mathrm{rpm}$.

*n.s. $(\mathrm{P}=0.1424)$

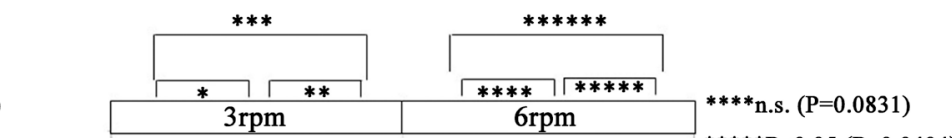

$* * \mathrm{P}<0.05(\mathrm{P}=0.118)$

$* * \mathrm{P}<0.05(\mathrm{P}=0.0002)$

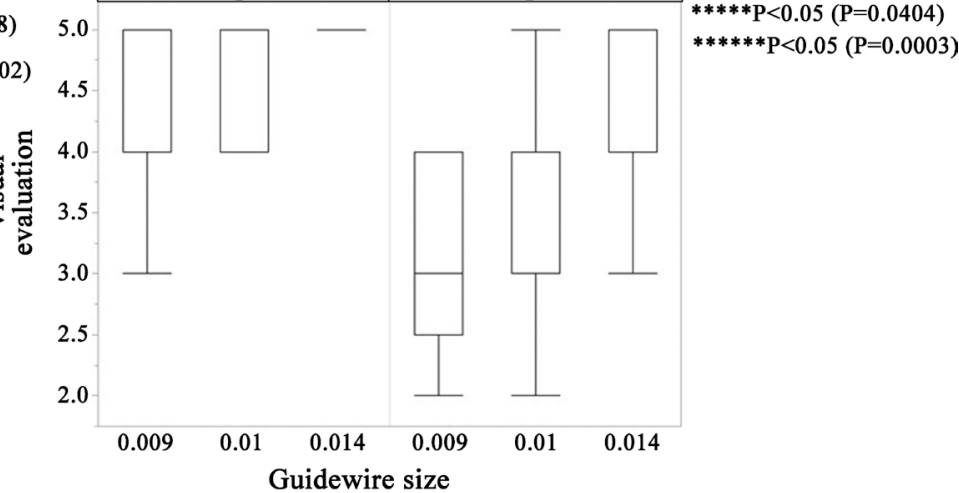

The differences do not reach statistically significant values. Results with Students-t test using the statistical analysis software Jamp1.4. \#\# In the figure: 6.0.

Figure 11. Box plots of differences in the visual evaluation results for the guide wires with whiskers. 
differences between the scores of the visual evaluations of the 0.009 and 0.010 -inch guide wires at 3.0 or $6.0 \mathrm{rpm}$. However, the visual evaluation scores of the 0.009- and 0.014-inch guide wires, and the 0.010- and 0.014-inch guide wires were statistically significantly different $(\mathrm{P}<0.05)$, with the 0.014 -inch guide wires showing statistically significantly lower visual evaluation scores $(\mathrm{P}<0.05)$.

For the visual evaluation of the differences in the rotation speeds of 3.0 and $6.0 \mathrm{rpm}$, the guide wires set horizontally and vertically showed statistically significant differences in visual evaluation scores (Figure 12), with the faster speed of rotation resulting in statistically significantly lower scores $(\mathrm{P}<.05)$. Further, there were no statistically significant differences in the scores of the different physicians who performed the visual evaluations in the study (Figure 13). The

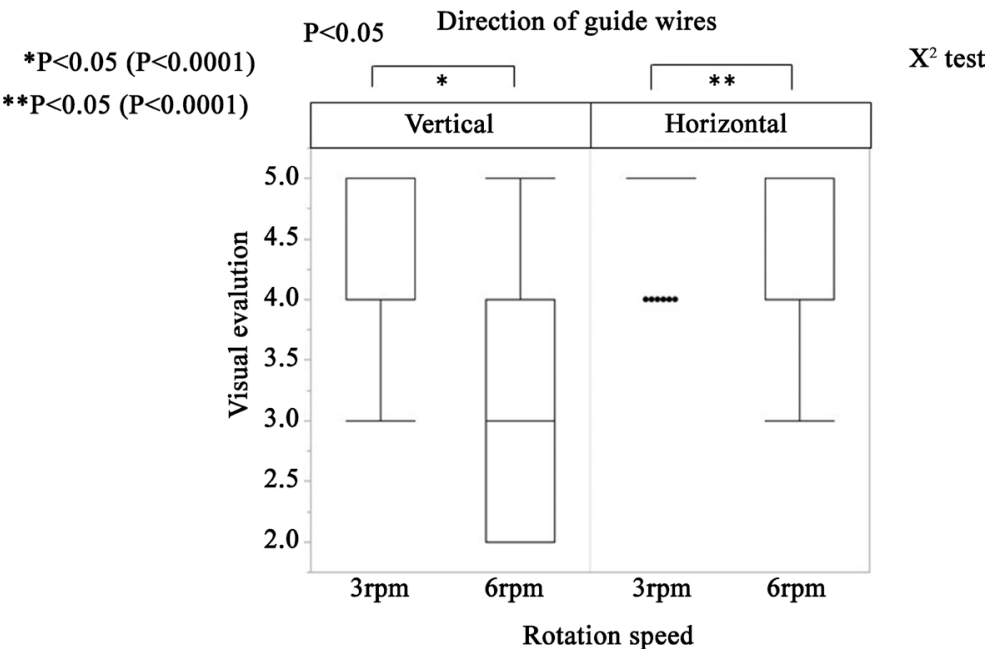

Results of $\mathrm{X}^{2}$ test using statistical analysis processing software Jamp1.4. \#\#In the figure: 3.0 and 6.0.

Figure 12. Boxplots of differences in the visual evaluation for different rotation speeds of horizontal and vertical guide wires. Statistical differences as indicated at the top of the figure.

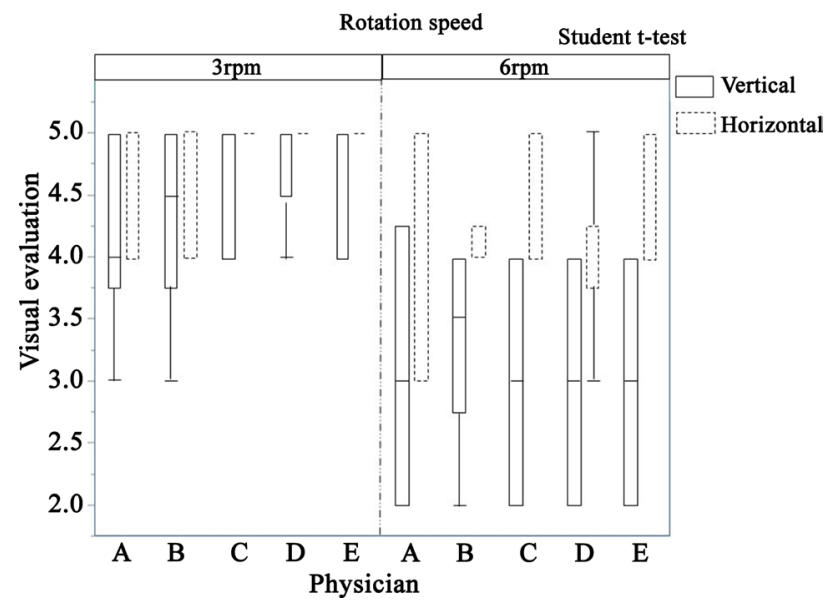

The differences do not reach statistically significant values. Results using Student- test using the statistical analysis software Jamp1.4

Figure 13. Box plots of differences in the visual evaluation results (with whiskers) by different physicians. 
results of the statistical analyses of the individual scores suggest that the differences in the thickness, direction, and speed of the guide wire movements may affect the visual evaluation at the set measurement geometry similarly to that experienced by the physicians who perform visual evaluations.

\subsection{Physical Evaluation with Guide Wires Set at the Different Orientations and Speeds}

When the guide wires of the same thickness were set horizontally and vertically, very similar profile curves were obtained at 3.0 and $6.0 \mathrm{rpm}$ (Figure 8). Further, the results showed similar changes for all the three guide wire diameters here. The changes in the profile curves of the guide wires with the different thicknesses, became smaller with thicker guide wires, at both speeds (Figure 8). The profile curves of the guide wires set horizontally and vertically showed no significant differences between the speeds of 3.0 and $6.0 \mathrm{rpm}$ (Figure 14). The moving speeds of the guide wires set horizontally and vertically on the KS dynamic phantom evaluated this time were $18.8 \mathrm{~mm} / \mathrm{sec}$ at $3.0 \mathrm{rpm}$, and $9.4 \mathrm{~mm} / \mathrm{sec}$ at $6.0 \mathrm{rpm}$. Images were recorded under the conditions where guide wires that rotated at these speeds were exposed to X-rays for an extremely short period with an X-ray output pulse width of $5 \mathrm{~ms}$. Based on the idea of Tanaka (2011) [1], when a guide wire moving at $18.8 \mathrm{~mm} / \mathrm{sec}$ is imaged for $5 \mathrm{~ms}$, the $0.094 \mathrm{~mm}$
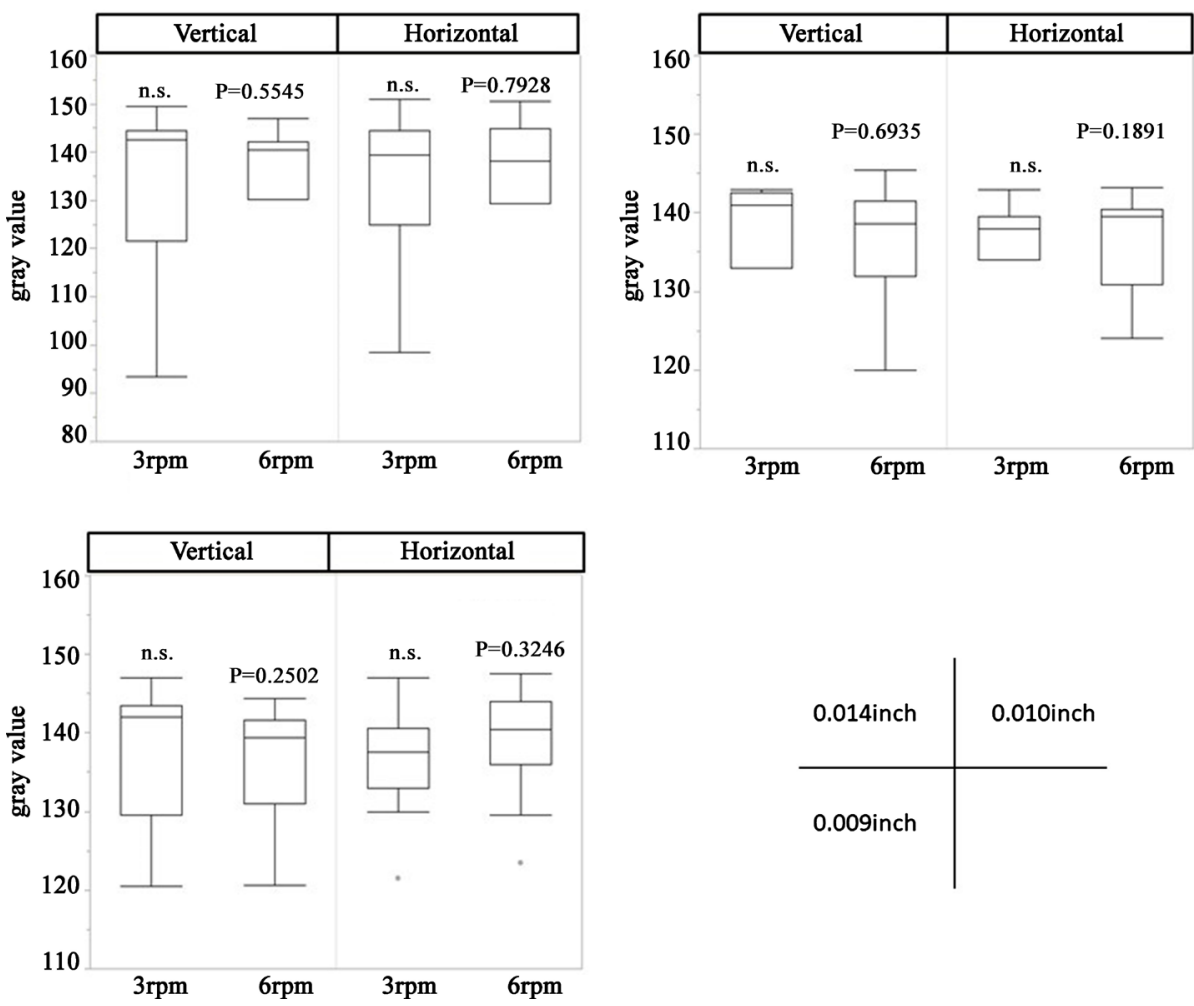

Differences are due to different orientations and speeds. Whiskers show standard deviations and the significance evaluations are at the top of the plots.

Figure 14. Boxplots of the 3.0 and $6.0 \mathrm{rpm}$ differences in the physical evaluation of the background noise with different guide wire diameters set horizontally and vertically. 
$\mathrm{X}$-ray images are blurred. Also, when a guide wire moving at $9.4 \mathrm{~mm} / \mathrm{sec}$ is imaged for $5 \mathrm{~ms}$, the blurring extends over $0.047 \mathrm{~mm}$. Taken together, these differences in the blurring between the guide wires set horizontally and vertically may not be physically expressed because both were clearly captured as static images with little blur due to the movement here.

\subsection{Evaluation of Background Noise with Guide Wires Moving at the Different Speeds}

There were no statistically significant differences in the background noise in the speeds (3.0 and $6.0 \mathrm{rpm}$ ) of the guide wires (Figure 15). This suggests that the background noise, which previous studies have reported to affect the image quality [4] [5] [6] [7], do not cause physical changes with the geometries compared in this study, clinically employed speeds of 3.0 and $6.0 \mathrm{rpm}$. There were statistically significant differences in the visual evaluations by physicians at any of the orientations and speeds of the guide wires set horizontally and vertically here. However, as the results of this physical evaluation suggest, the differences may arise due to the human eye rather than due to background noise.

\section{Conclusions}

Using a KS dynamic phantom (Figure 1) by Kyoto Kagaku Co., this study conducted visual and physical evaluations of differences that were visible by changes in the orientation and speed of movement of guide wires set horizontally and vertically. The results showed a lower visual evaluation only in the vertical direction at the more rapid movement here, but this did not result in significant changes in the physical evaluation. This suggests that the structure and characteristics of the human eyes would be involved, as human vision is stronger with lateral movements and weaker with vertical movements due to the alignment of the human eyes, side by side [8] [9] [10] [11] [12]. Further, the 3.0 and $6.0 \mathrm{rpm}$ speeds that were used here are only a part of the speed range used in clinical

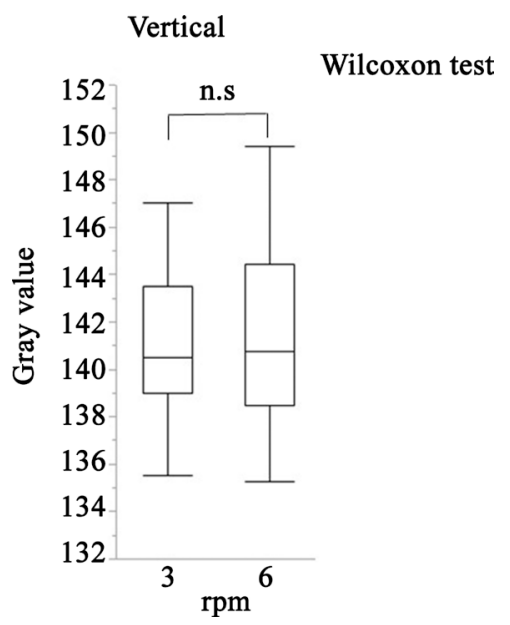

Figure 15. Boxplots of the 3.0 and $6.0 \mathrm{rpm}$ differences in the physical evaluation for the background noise with 0.009 -inch thick vertical guide wires. 
applications. The findings have shown that the deterioration in visibility due to differences in the orientation of the guide wires, a therapeutic device commonly used in clinical settings, is not due to the image quality, but that it is greatly influenced by the human eye observing it.

Findings from this basic study can be utilized to improve the visibility in the $\mathrm{X}$-ray moving images by paying attention to the observation environment of the observer of the X-ray moving images. In addition, the findings of this study can also be used to determine protocols for improving visibility in X-ray moving images, such as adjusting the X-ray dose in an X-ray device when further improvement is required. Therefore, this study was able to provide suggestions to contribute to the development of improved visibility of X-ray moving images.

\section{Acknowledgements}

We wish to express our gratitude to the physicians of the Cardio Vascular Center and radiological technologists of the Radiation Technology Division of Showa University for cooperation in data collection and image evaluations for this study. The authors have no conflicts of interest to declare.

\section{Conflicts of Interest}

The authors declare no conflicts of interest regarding the publication of this paper.

\section{References}

[1] Tanaka, R., Shiraishi, J., Takamori, M. and Watari, C. (2011) ROC Analysis for Evaluating the Detectability of Image Unsharpness due to the Patient's Movement: Phantom Study Comparing Preview and Diagnostic LCDs. Jpn. J. Radiol. Technol, 67, 772-778. https://doi.org/10.6009/jjrt.67.772

[2] Saragusti, I., Karasik, A., Sharon, I. and Smilansky, U. (2005) Quantitative Analysis of Shape Attributes Based on Contours and Section Profiles in Artifact Analysis. Journal of Archaeological Science, 32, 841-853. https://doi.org/10.1016/j.jas.2005.01.002

[3] Sato, H., Kittaka, D., Ohsawa, M. and Kato, K. (2019) A Study on Fluoroscopic Images in Exposure Reduction Techniques-Focusing on the Image Quality Of Fluoroscopic Images and Exposure Images. Journal of Applied Clinical Medical Physics, 20, 125-131. https://doi.org/10.1002/acm2.12549

[4] Söderman, M., Mauti, M., Boon, S., et al. (2013) Radiation Dose in Neuroangiography Using Image Noise Reduction Technology: A Population Study Based on 614 Patients. Interventional Neuroradiology, 55, 1365-1372. https://doi.org/10.1007/s00234-013-1276-0

[5] Nakamura, S., Kobayashi, T., Funatsu, A., et al. (2016) Patient Radiation Dose Reduction Using an X-Ray Imaging Noise Reduction Technology for Cardiac Angiography and Intervention. Heart and Vessels, 31, 655-663. https://doi.org/10.1007/s00380-015-0667-Z

[6] Kayikcioglu, T., Gangal, A., Turhal, M. and Kose, C. (2002) A Surface Based Method for Detection of Corronary Boundaries on Poor Quality X-Ray Angiogram Images. Pattern Recognition Letters, 23, 783-802. 
https://doi.org/10.1016/S0167-8655(01)00156-8

[7] Yadava, G.K., Kuhls-Gilcrist, A.T., Rudin, S., Patel, V.K., Hoffmann, K.R. and Bednarek, D.R. (2008) A Practical Exposure-Equivalent Metric for Instrumentation Noise in X-Ray Imaging Systems. Physics in Medicine \& Biology, 53, 5107-5121. https://doi.org/10.1088/0031-9155/53/18/017

[8] Igawa, M., Nakayama, N. and Maeda, F. (2006) Comparison of Eye Movements While Reading Vertically/Horizontally Arranged Words: Special Feature, 59th Annual Meeting of the Japanese Society of Clinical Ophthalmology (5). Japanese Journal of Clinical Ophthalmology, 60, 1251-1255.

[9] Arase, M. (2007) Visual Expressions for Editors and Designers. In: Introduction to Editorial Design, 11th Edition, Media Pal, Tokyo, Japan, 1-6.

[10] Iwashita, T. (1965) Readability of Telop Characters. NHK Institute of Broadcasting Research, 19, 484-491. https://doi.org/10.3169/itej1954.19.484

[11] Yamakawa, K., Itoh, K., Yumoto, M., Uno, A. and Kaga, K. (2004) Magnetoencephalographic Study on the Recognition Potential: Responses to Vertically and Horizontally Arranged Words and Visual Stimuli. Technical Report of IEICE, 103 , 19-24.

[12] Kajii, N. and Osaka, N. (2000) Optimal Viewing Position in Vertically and Horizontally Presented Japanese Words. Perception \& Psychophysics, 62, 1634-1644. https://doi.org/10.3758/BF03212161 